\title{
SUBHARMONICITY OF THE LYAPONOV INDEX
}

\author{
W. CRAIG AND B. SIMON*
}

1. Introduction. There has been intense current interest in a class of one dimensional Schrödinger operators

$$
\frac{-d^{2}}{d x^{2}}+V_{\omega}(x)
$$

on $L^{2}(-\infty, \infty)$ and their discrete analogs on $l^{2}(Z)$

$$
(M u)(n)=u(n+1)+u(n-1)+V_{\omega}(n) u(n)
$$

where the potential $V$ is an ergodic process in the sense that the index $\omega$ lies in a probability measure space $\left(\Omega, d \mu_{0}\right)$ which supports a group $\tau_{x}(x \in R$ in case (1.1) or $x \in Z$ in case (1.2)) of measure preserving ergodic transformations with $V_{\omega}(x+y)=V_{\tau_{y} \omega}(x)$, where $\sup \left\{\left|V_{\omega}(x)\right| \mid x \in R\right.$ or $\left.Z, \omega \in \Omega\right\}<\infty$. The most heavily studied cases are the "random" ones where $\tau_{x}$ has strong mixing properties (e.g., i.i.d.'s in case $(1.2)$ [8, 3] or Morse functions composed with Brownian motion on a compact manifold in case $(1.3)[4,9,2])$ and the almost periodic case where $\Omega$ is a compact metric space and the $\tau$ 's are isometric (see [12] for a review of this).

The present paper represents a contribution to this theory. Motivated in part by old work of Thouless [13], and in part by recent work of Hermann [5] (see below), we will prove that a basic quantity is a subharmonic function, and more significantly, derive some important consequence of this observation. Interestingly enough, the fact that certain functions are upper semicontinuous while others are not will play a major role. For this reason, we single out functions which are subharmonic except for semicontinuity:

Definition. A function, $f$, on $C$ with values in $[-\infty, \infty)$ is called submean if and only if for all $z_{0} \in C$ and $r>0$ we have that

$$
f\left(z_{0}\right) \leqslant(2 \pi)^{-1} \int_{0}^{2 \pi} f\left(z_{0}+r e^{i \theta}\right) d \theta .
$$

For the reader's convenience we recall

Definition. A function $f$ on $C$ is called uppersemicontinuous (u.s.c.) if and only if for any $z_{n} \rightarrow z_{\infty}, \varlimsup_{\lim } \rightarrow \infty f\left(z_{n}\right) \leqslant f\left(z_{\infty}\right)$. Equivalently, if given $z_{\infty}$ and $\epsilon$ we can find $\delta$ with $f(z)<f\left(z_{\infty}\right)+\epsilon$ if $\left|z-z_{\infty}\right|<\delta$.

Received September 20, 1982.

* Research partially supported by USNSF under grant MCS-81-20833. 
Definition. A function $f$ is called subharmonic if and only if it is submean and u.s.c.

Uppersemicontinuity is singled out because it implies a strong form of the maximum principle. For our purposes it is relevant because:

THEOREM 1.1. If $f$ is subharmonic and $z_{0}$ is fixed then

$$
f\left(z_{0}\right)=\lim _{r \rightarrow 0}\left(\pi r^{2}\right)^{-1} \int_{\left|z-z_{0}\right| \leqslant r} f(z) d^{2} z
$$

and if $f$ is submean

$$
f\left(z_{0}\right) \leqslant \lim _{r \rightarrow 0}\left(\pi r^{2}\right)^{-1} \int_{\left|z-z_{0}\right| \leqslant r} f(z) d^{2} z
$$

Proof. (1.5) is an immediate consequence of (1.3). The other half of (1.4) follows by u.s.c.

For the reader's convenience we also recall:

THEOREM 1.2. If $f_{n}(z)$ is a sequence of submean functions with $\sup _{|z|<R}\left|f_{n}(z)\right|$ $<\infty$ for any $R$, then $f_{\infty}(z) \equiv \varlimsup \lim f_{n}(z)$ is submean.

Proof. For any $N$, obviously

$$
f_{n}\left(z_{0}\right) \leqslant(2 \pi)^{-1} \int f_{n}\left(z_{0}+r e^{i \theta}\right) d \theta \leqslant(2 \pi)^{-1} \int \sup _{n \geqslant N} f_{n}\left(z_{0}+r e^{i \theta}\right) d \theta
$$

so $\sup _{n \geqslant N} f_{n}\left(z_{0}\right)$ is submean. By the monotone convergence then, $\inf _{N} \sup _{n \geqslant N} f_{n}$ $\equiv f_{\infty}$ is submean.

THEOREM 1.3. If $f_{n}$ is a decreasing family of subharmonic functions then $f_{\infty}(z)=\inf _{n} f_{n}(z)$ is subharmonic.

Proof. $f_{\infty}$ is submean by the last theorem. An inf of u.s.c, functions is u.s.c.

We will also need the following standard theorem (see e.g., [10]):

THEOREM 1.4. If $A(z)$ is an entire matrix valued function, the $\log \|A(z)\|$ is subharmonic.

In the context of equations (1.1) and (1.2) define the $2 \times 2$ matrix $T_{l}(\omega, E)$ so that in case $(1.1) T_{l}(\omega, E)(a, b)$ is $\left(u(l), u^{\prime}(l)\right)$, where $u$ solves $(1.1) u=E u$ with $u(0)=a, u^{\prime}(0)=b$. In case $(1.2)$, let $T_{l}(\omega, E)(a, b)$ be $(u(l+1), u(l))$ where $u(1)=a, u(0)=b$. We define

$$
\gamma_{l}(\omega, E)=|l|^{-1} \ln \left\|T_{l}(\omega, E)\right\|
$$

The subadditive ergodic theorem [11] asserts that 
THEOREM 1.5. $\gamma(E)=\lim _{|l| \rightarrow \infty} \int_{\Omega_{0}} d \mu_{0}(\omega) \gamma_{l}(E, \omega) \equiv \inf _{l} \int d \mu_{0}(\omega) \gamma_{l}(E, \omega)$ exists, and for $E$ fixed and a.e. $\omega, \gamma_{l}(\omega, E) \rightarrow \gamma(E)$.

$\gamma(E)$ is called the Lyaponov exponent. Please note the difference between $\gamma(E)$ and $\gamma(E, \omega)$; it is the former, which is an averaged quantity, which is considered in most of this work. Our basic observation, whose consequences we will develop, will appear in section 2 :

THEOREM 2.1. $\gamma(E)$ is subharmonic.

A basic consequence will be that if we define

$$
\bar{\gamma}(E, \omega)=\varlimsup_{|l| \rightarrow \infty} \gamma_{l}(\omega, E)
$$

then

THEOREM 2.3. For a.e. $\omega$, we have for all $E$

$$
\bar{\gamma}(E, \omega) \leqslant \gamma(E) .
$$

In the almost periodic case, a.e. $\omega$ can be replaced by all $\omega$.

Using rather different methods that appear special to the a.p. case, Johnson [6] has proven Thm. 2.3 in the a.p. case.

There is a connection between Theorem 2.1 and the fact that the spectral radius of a Banach algebra valued analytic function is subharmonic. This fact, and related results, are discussed in $[14,15,16]$.

In section 3, we will use Thm. 2.3 to prove:

THEOREM 3.2. If a.e. $\omega$, we have that any solution $u$ of (1.1) $u=E u$ (resp. (1.2) $u=E u)$ obeys

$$
\begin{array}{r}
\varliminf_{|l| \rightarrow \infty} l^{-1} \ln \left[|u(l)|^{2}+\left|u^{\prime}(l)\right|^{2}\right]^{1 / 2} \geqslant-\gamma(E) \\
\text { resp. } \left.\varliminf_{|l| \rightarrow \infty} l^{-1} \ln \left[|u(l)|^{2}+|u(l-1)|^{2}\right]^{1 / 2} \geqslant-\gamma(E)\right) .
\end{array}
$$

This result has an important consequence in the Brownian model of random motion. In this model, $\left(\Omega, d \mu_{0}\right)$ is two-sided Brownian motion on a compact Riemannian manifold, $M$, with Brownian path $b(t) ; f$ is a Morse function on $M$ and $V_{\omega}(x)=f(b(x))$. In [4], Goldsheid et al. proved that for a.e. $\omega(1.1)$ had only (dense) point spectrum and in [9], Molchanov proved that for a.e. $\omega$, every eigenfunction decays exponentially.

In section 4, we simplify the proof of the Thouless formula given by Avron-Simon [1], and prove it for all $E$, and in section 5, we prove the following theorem on the modulus of continuity of the density of states. 
Definition. A function is log-Hölder continuous if for all $R$, there is a $C>0$, such that whenever $|x|<R,|x-y|<\frac{1}{2}$, then

$$
|f(x)-f(y)| \leqslant c\left(\ln |x-y|^{-1}\right)^{-1} .
$$

THEOREMS 5.1, 5.2. In both cases (1.1) and (1.2), the integrated density of states is log-Hölder continuous.

The fact that $k(E)$ is uniformly equicontinuous allows us to conclude that whenever $k(E)$ or $\int d \mu_{0}(\omega) k_{\omega}(E)$ converges pointwise (see Avron-Simon [1]), the convergence is actually uniform on compact sets.

Our realization of the importance of subharmonicity comes from two sources. First, the integral

$$
\int \ln \left|E-E^{\prime}\right| d k\left(E^{\prime}\right)
$$

occurs in the Thouless formula, while

$$
\gamma_{l}(E)=\frac{1}{l} \ln \left\|T_{l}(E)\right\|
$$

and both these quantities look suggestively subharmonic. Secondly, M. Hermann [5] studied a situation in which $T_{l}(E, \omega)$ for $E$ fixed was analytic in $\omega$ and for which the integrals over $d \mu_{0}(\omega)$ were averages over the circle, so the submean property was very useful. While semicontinuity played no role in his work, and while he used only subharmonicity in $\omega$, his considerations were extremely useful to us.

It is a pleasure to thank J. Avron for valuable discussions.

\section{Basic results.}

THEOREM 2.1. $\gamma(E)$ is subharmonic.

Proof. By the inequality $\|A B\| \leqslant\|A\|\|B\|$, we have that $(l+m) \gamma_{l+m}(E, \omega)$ $\leqslant l \gamma_{l}(E, \omega)+m \gamma_{m}\left(E, T^{l} \omega\right)$, so averaging over $\omega$, the quantity $l \gamma_{l}(E) \equiv \int l \gamma_{l}(E, \omega)$ $d \mu_{0}(\omega)$ is subadditive and thus $\gamma(E)=\inf \gamma_{2^{\prime}}(E)$ and $\gamma_{2^{\prime}}(E)$ is monotone decreasing. By Thm. 1.4, $\gamma_{l}(E)$ is subharmonic, so by Thm. 1.5, so is $\gamma(E)$.

THEOREM 2.2. $\bar{\gamma}(E, \omega)$ is submean.

Proof. By Thm. 1.4, $\gamma_{l}(E, \omega)$ is subharmonic and so submean. Thus, this result follows from $\mathrm{Thm}$. 1.2.

THEOREM 2.3. For a.e. $\omega$, we have that for all $E$

$$
\bar{\gamma}(E, \omega) \leqslant \gamma(E) .
$$

In the almost periodic case, a.e. $\omega$ can be replaced by all $\omega$. 
Proof. Fix $E$. By Thm. 1.5, $\bar{\gamma}(E, \omega)=\gamma(E)$ for a.e. $\omega$, so $\bar{\gamma}(E, \omega)=\gamma(E)$ for a.e. pairs $(\omega, E)$ (with respect to $\left.d \mu_{0} \times d^{2} E\right)$. Thus, by Fubini's theorem, for a.e. $\omega, \bar{\gamma}(E, \omega)=\gamma(E)$ for a.e. $E$. In the a.p. case, this holds for all $\omega$ (and all $E$ with $\operatorname{Im} E>0$ ) by the proof of the Thouless formula (see [1] or section 4 below). If $\bar{\gamma}(E, \omega)=\gamma(E)$ for a.e. $E$, and $E_{0}$ is fixed, we have for any $E_{0}$

$$
\int_{\left|E-E_{0}\right| \leqslant r} \bar{\gamma}(E, \omega) d^{2} E=\int_{\left|E-E_{0}\right| \leqslant r} \gamma(E) d^{2} E .
$$

Divide by $\left(\pi r^{2}\right)$ and take $r$ to zero. The right side converges to $\gamma\left(E_{0}\right)$ by Thms. 2.1 and 1.1, and the left side is larger than $\bar{\gamma}(E, \omega)$ by Thms. 2.2 and 1.1.

3. Lower bounds on eigenfunction decay. We have already defined $\bar{\gamma}(E, \omega)$. Define $\underline{\gamma}(E, \omega)$ to be $\underline{\lim }$. Given a solution of (1.1) $u=E u$ (resp. (1.2) $u=E u$ ) let $\Phi_{l}$ be the two vector $\left(u(l), u^{\prime}(l)\right)$ (resp. $(u(l+1), u(l))$ and let $\bar{u}_{ \pm}$ $\equiv \overline{\lim }_{l \rightarrow \pm \infty}|l|^{-1} \ln \left\|\Phi_{l}\right\|$ and $\underline{u}_{ \pm}=\underline{\lim }_{l \rightarrow \pm \infty}|l|^{-1} \ln \left\|\Phi_{l}\right\|$. Then:

Theorem 3.1. Normalize $u$, so $\left\|\Phi_{0}\right\|=1$. Then

$$
\left\|\Phi_{l}\right\|\left\|T_{l}\right\| \geqslant 1
$$

so that

$$
\underline{\gamma}+\bar{u}_{ \pm} \geqslant 0, \quad \bar{\gamma}+\underline{u}_{ \pm} \geqslant 0
$$

Proof. (3.2) follows from (3.1) by taking logs, dividing by $l$ and taking $l \rightarrow \infty$ through a suitable subsequence. Let $J=\left(\begin{array}{rr}0 & -1 \\ 1 & 0\end{array}\right)$. Since $T_{l}$ has determinant 1 (constancy of Wronskian),

$$
\left(J T_{l} J^{-1}\right)^{t}=T_{l}^{-1}
$$

where $t$ is transpose, so since $\|J\|=1$, we have that $\left\|T_{l}\right\|=\left\|T_{l}^{-1}\right\|$. Therefore

$$
1=\left\|\Phi_{0}\right\|=\left\|T_{l}^{-1} \Phi_{l}\right\| \leqslant\left\|T_{l}\right\|\left\|\Phi_{l}\right\|
$$

As an immediate consequence of this theorem and Theorem 2.3:

THEOREM 3.2. For any solution $u, \underline{u}_{ \pm} \geqslant-\gamma$.

This implies

THEOREM 3.3. For any solution $u$, if $\bar{u}_{+} \leqslant-\gamma$ and $\bar{u}_{-} \leqslant-\gamma$, then $\bar{u}_{ \pm}=\underline{u}_{ \pm}=-\gamma$ and $\bar{\gamma}=\underline{\gamma}=\gamma$.

Proof. We have $-\gamma \geqslant \bar{u}_{+} \geqslant \underline{u}_{ \pm} \geqslant-\gamma$ by the last two theorems, and then by (3.2) and Thm. $2.3 \gamma \leqslant \underline{\gamma} \leqslant \bar{\gamma} \leqslant \gamma$.

In the Brownian model, Carmona [2] has proven that for a.e. $\omega$, we have that for every eigenvalue $E$, there is an eigenfunction $u_{E}$ with $u_{ \pm} \leqslant-\gamma$ (and these 
eigenfunctions are complete by [4]). Thus

THEOREM 3.4. In the Brownian model, for a.e. $\omega$ and every eigenfunction, eigenvalue pair $(u, E)$ we have

$$
\lim _{|l| \rightarrow \infty} l^{-1} \ln \left\|\Phi_{l}\right\| \text { and } \lim _{|l| \rightarrow \infty} l^{-1} \ln \left\|T_{l}(E)\right\|
$$

exist; the first equals $-\gamma(E)$ and the second equals $\gamma(E)$.

Remark. We are only asserting $|l|^{-1} \ln \left\|T_{l}(E)\right\|$ has a limit for all eigenvalues $E$ of $H(\omega)$, not for all $E$.

4. The Thouless formula. In [13], Thouless discussed a formula relating $\gamma$ and the integrated density of states, $k$, in the case (1.2):

$$
\gamma(E)=\int \ln \left|E-E^{\prime}\right| d k\left(E^{\prime}\right)
$$

Thouless' proof was formal at some points, and as noted by Avron-Simon [1], there are examples where the spectral measure of $M_{\omega}$ is supported on the set of $E$ for which either $\bar{\gamma} \neq \underline{\gamma}$ or $\bar{\gamma}(E, \omega) \neq \int \ln \left|E-E^{\prime}\right| d k\left(E^{\prime}\right)$. They give a rigorous proof for (4.1) for a.e. $E$ using some functional analytic gymnastics. The first step is that there is a sequence of measures on $(-A, A)$ with $A=2+\|V\|_{\infty}$, called $k_{l}$, with $\mid d k_{l} \rightarrow d k$ weakly (a.e. $\omega$ in the general case, all $\omega$ in the a.p. case) with

$$
\gamma_{l}(E, \omega)=\int \ln \left|E-E^{\prime}\right| d k_{l}\left(E^{\prime}\right)
$$

If $E \notin[-A, A]$ ( $E$ may be complex), the $\ln \left|E-E^{\prime}\right|$ is continuous for $E^{\prime} \in[-A, A]$ and (4.1) follows. The gymnastics in [1] were required to handle $E \in[-A, A]$. To give a simpler proof, we note:

LEMMA 4.1. Define

$$
\int \ln \left|E-E^{\prime}\right| d k\left(E^{\prime}\right)
$$

by the convention that it is $-\infty$ if the integral diverges to $-\infty$. Then it is a subharmonic function.

Proof. $\ln \left|\cdot-E^{\prime}\right|$ is subharmonic, so the expression is clearly submean. For $a>0$, define

$$
f_{a}(E)=\int \max \left\{\ln \left|E-E^{\prime}\right|,-a\right\} d k\left(E^{\prime}\right)
$$

Then $f_{a}$ is continuous and the expression is just $\inf _{a>0} f_{a}(E)$ by the monotone convergence theorem. Thus the expression is upper semicontinuous.

A corollary of Thm. 1.1 is that if the subharmonic functions agree a.e. in the complex plane, they agree everywhere. Thus, knowing (4.1) for $\operatorname{Im} E \neq 0$ (which is easy, see [1]), we find that by combining Lemma 4.1 with Thm. 2.1: 
THEOREM 4.2. (4.1) holds for all $E$.

In the continuous case (1.1), one must compare $\gamma(E)$ with the free Lyaponov exponent $\gamma_{0}(E)$. Define, for $E \in \mathrm{C}, \gamma_{0}(E)=\operatorname{Re}(\sqrt{-E})$, where the branch is chosen so that $\sqrt{-E}>0$ for $E<0$. Let $k_{0}(E)=(1 / \pi) \sqrt{\max \{0, E\}}$. It is shown in [1] that for $\operatorname{Im} E \neq 0$

$$
\gamma(E)-\gamma_{0}(E)=\int \ln \left|E-E^{\prime}\right|\left\{d k\left(E^{\prime}\right)-d k_{0}\left(E^{\prime}\right)\right\}
$$

The integral on the right is conditionally convergent in the sense that it is proven that

$$
\lim _{a \rightarrow \infty} \int_{-\infty}^{a} \ln \left|E-E^{\prime}\right| d\left(k-k_{0}\right)\left(E^{\prime}\right)
$$

exists and is finite if $\operatorname{Im} E \neq 0$. Similarly, we find

$$
\gamma(E)-\gamma_{0}(E+a)=\int \ln \left|E-E^{\prime}\right|\left\{d k\left(E^{\prime}\right)-d k_{0}\left(E^{\prime}+a\right)\right\} .
$$

The integral $\int_{a}^{\infty} \ln \left|E-E^{\prime}\right|\left\{d k\left(E^{\prime}\right)-d k_{0}\left(E^{\prime}+a\right)\right\}$ is harmonic on $\mathrm{C}-[a, \infty)$, hence if the integral in (4.3) is defined to be $-\infty$ whenever it diverges to $-\infty$, then the right side is subharmonic on $\mathrm{C}-[a, \infty)$. As before, this establishes (4.3) and then (4.2) for all $E$.

The Thouless formula for all $E$ implies several general principles:

(a) Since $\ln \left|E-E^{\prime}\right|$ is harmonic away from $E^{\prime}$, and $\operatorname{supp}(d k)=\operatorname{spec}\left(H_{\omega}\right)$ we see that $\gamma(E)$ is harmonic away from $\operatorname{spec}\left(H_{\omega}\right)$.

(b) Using (a), Johnson [6] proves in the a.p. case that for any open interval $I \subset R$, either $I \cap \operatorname{spec}\left(H_{\omega}\right)$ is empty or it has strictly positive logarithmic capacity. Using his proof and our arguments to establish (a), this result is true in the general ergodic case.

(c) Since $\gamma$ is u.s.c. and nonnegative, at points with $\gamma(E)=0$ (necessarily this implies that $\left.E \in \operatorname{spec}\left(H_{\omega}\right)[1]\right), \gamma$ is continuous.

(d) Since $\ln \left|E+i \epsilon-E^{\prime}\right|$ decreases monotonically to $\ln \left|E-E^{\prime}\right|$ as $\omega \downarrow 0$ (when $E, E^{\prime}$ are real), we see that for any real $E, \gamma(E)=\lim _{\epsilon \downarrow 0} \gamma(E+i \epsilon)$. This is how Johnson [6] defines $\gamma$ for $E$ real. (He doesn't appear to note that $\gamma$ defined this way is the a.e. Lyaponov exponent.)

(e) If $E<E^{\prime}$, then $\ln \left|E-\epsilon-E^{\prime}\right|$ converges monotonically to $\ln \left|E-E^{\prime}\right|$ and if $E^{\prime}<E-\epsilon_{0}$, then as $\epsilon \downarrow 0, \ln \left|E-\epsilon-E^{\prime}\right|$ is bounded, so if $(a, b)$ is disjoint from $\operatorname{spec}\left(H_{\omega}\right)$ but $b \in \operatorname{spec}\left(H_{\omega}\right)$ we have that $\gamma(b)=\lim _{\epsilon \downarrow 0} \gamma(b-\epsilon)$. This is a result of Johnson [6] in the a.p. case.

5. Log-Hölder continuity of the integrated density of states. In [1], [7], it is a basic result that $k(E)$ is a continuous function of $E$, but the proof gives no estimate on the modulus of continuity. We want to note that the Thouless formula combined with the nonnegativity of $\gamma$ implies a continuity of $k$ which is uniform for $E$ in compact sets and uniform in $V$ as $V$ runs through sets with 
$\|V\|_{\infty}$ bounded. We consider both the case where $k(E)$ is the density of states and the case where we average over an auxiliary parameter such as occurs for $V(n)=\cos (2 \pi \alpha n+\theta)$ where $\alpha$ is rational and $\theta$ is averaged. The proof of $\log$-Hölder continuity is identical in these two cases, but the discrete case (1.2) is slightly different from the continuous case (1.1); they are treated in Theorems 5.1 and 5.2 respectively.

THEOREM 5.1. In case (1.2) let $E_{0}$ and $E_{1}$ be real with $\left|E_{0}-E_{1}\right|<\frac{1}{2}$. Then

$$
\left|k\left(E_{1}\right)-k\left(E_{0}\right)\right| \leqslant \ln \left[\left|E_{1}\right|+\left|E_{0}\right|+\|V\|_{\infty}+2\right] / \ln \left\{\left|E_{0}-E_{1}\right|^{-1}\right\} .
$$

Proof. Without loss of generality, assume $E_{1}>E_{0}$.

$$
\begin{aligned}
0 \leqslant & \gamma\left(E_{0}\right)=\int \ln \left|E_{0}-E^{\prime}\right| d k\left(E^{\prime}\right) \\
= & \int_{E_{0}}^{E_{1}} \ln \left|E_{0}-E^{\prime}\right| d k\left(E^{\prime}\right) \\
& +\int_{\substack{\left|E_{0}-E^{\prime}\right| \leqslant 1 \\
\left\{E^{\prime}<E_{0}\right\} \cup\left\{E_{1}<E^{\prime}\right\}}} \ln \left|E_{0}-E^{\prime}\right| d k\left(E^{\prime}\right)+\int_{1 \leqslant\left|E_{0}-E^{\prime}\right|} \ln \left|E_{0}-E^{\prime}\right| d k\left(E^{\prime}\right) . \\
&
\end{aligned}
$$

Hence, since the second integral is negative

$$
\begin{aligned}
-\ln \left|E_{1}-E_{0}\right| \int_{E_{0}}^{E_{1}} d k\left(E^{\prime}\right) & \leqslant \int_{1 \leqslant\left|E_{0}-E^{\prime}\right|} \ln \left|E_{0}-E^{\prime}\right| d k\left(E^{\prime}\right) \\
& \leqslant \ln \left\{\left|E_{0}\right|+\|V\|_{\infty}+2\right\}
\end{aligned}
$$

In the continuous case (1.1), we again use a comparison with the free case. For $E>-\|V\|_{\infty}, \gamma_{0}\left(E+\|V\|_{\infty}\right)=0$, hence for $\omega$ such that (4.2) holds,

$$
0 \leqslant \gamma\left(E_{0}\right)-\gamma_{0}\left(E_{0}+\|V\|_{\infty}\right)=\int \ln \left|E_{0}-E^{\prime}\right|\left\{d k\left(E^{\prime}\right)-d k_{0}\left(E^{\prime}+\|V\|_{\infty}\right)\right\} .
$$

Take any $E_{1}$ so that $\left|E_{1}-E_{0}\right|<\frac{1}{2}$, again $E_{1}>E_{0}$,

$$
\begin{aligned}
0 \leqslant & \int_{E_{0}}^{E_{1}} \ln \left|E_{0}-E^{\prime}\right| d k\left(E^{\prime}\right)+\int_{\substack{1 \leqslant\left|E_{0}-E^{\prime}\right| \\
E^{\prime}<E_{0}+1}} \ln \left|E_{0}-E^{\prime}\right| d k\left(E^{\prime}\right) \\
& -\int_{\left|E_{0}-E^{\prime}\right|<1} \ln \left|E_{0}-E^{\prime}\right| d k_{0}\left(E^{\prime}+\|V\|_{\infty}\right) \\
& +\int_{E_{0}+1<E^{\prime}} \ln \left|E_{0}-E^{\prime}\right|\left\{d k\left(E^{\prime}\right)-d k\left(E^{\prime}+\|V\|_{\infty}\right)\right\} .
\end{aligned}
$$

Using that, [1]

$$
\left|k\left(E^{\prime}\right)-k_{0}\left(E^{\prime}+\|V\|_{\infty}\right)\right| \leqslant D\left(\left|E^{\prime}\right|+1\right)^{1 / 2}
$$


we find that

$$
\int_{E_{0}}^{E_{1}} d k(E) \leqslant \tilde{D}\left\{\ln \left|E_{0}-E_{1}\right|^{-1}\right\}^{-1}
$$

where $\tilde{D}$ depends only on $\left|E_{0}\right|$ and $\|V\|_{\infty}$. Thus we have shown

THEOREM 5.2. In the case (1.1), for any $a, b>0$ there exists a $D$ such that

$$
\left|k\left(E_{1}\right)-k\left(E_{0}\right)\right| \leqslant D\left\{\ln \left|E_{1}-E_{0}\right|^{-1}\right\}^{-1}
$$

for all $V$ with $\|V\|_{\infty}<a$, and all $E_{1}, E_{0}$ with $E_{0}<b,\left|E_{1}-E_{0}\right|<\frac{1}{2}$.

In [1], Avron-Simon proved pointwise in $E$ convergence of $k(E)$ or $\int k_{0}(E) d \theta$ in certain situations. By the last two theorems, in all of these situations one has equicontinuity in $E$, hence:

THEOREM 5.3. The various pointwise convergence results on $k$ in [1] (as frequency models vary) can be replaced by convergence uniform in $E$ as $E$ runs through compacts.

We want to note a further continuity result:

THEOREM 5.4. In the case where [1] proves pointwise convergence on $k$, one has for any $E$, upper-semicontinuity in $\gamma(E)$.

Remarks. 1. For example, in (1.2), if $V_{n}(x)=f\left(\alpha_{n} x+\theta_{n}\right)$ with $f$ continuous on the circle and $\alpha_{n} \rightarrow \alpha$ irrational, we claim that $\varlimsup_{\lim } \gamma\left(E_{n}, \alpha_{n}\right) \leqslant \gamma(E, \alpha)$ if $E_{n} \rightarrow E$.

2. There are examples where $\lim _{\gamma}\left(E_{n}, \alpha_{n}\right)<\gamma(E, \alpha)$. For take the case $V_{n}(x)=3 f\left(\alpha_{n} x+\theta_{n}\right)$ and $E \in \operatorname{spec}\left(H_{\alpha}\right)$. We confine $\theta_{n}, E_{n}$ so $E_{n} \in \operatorname{spec}\left(H\left(\alpha_{n}\right.\right.$, $\left.\theta_{n}\right)$ ) [1] and $E_{n} \rightarrow E$. Then $\gamma\left(E_{n}, \alpha_{n}\right)=0$ (since $H\left(\alpha_{n}, \theta_{n}\right)$ is periodic), but $\gamma(E, \alpha) \geqslant \ln (3 / 2)[1]$.

Proof. $\gamma_{l}(E)$ is continuous for finite $l$, so we need only use $\gamma(E)$ $=\inf _{n} \gamma_{2^{n}}(E)$.

\section{REFERENCES}

1. J. Avron, AND B. Simon, Almost periodic Schrödinger operators, II. The integrated density of states, Duke Math. J. 50 (1983), 369-391.

2. R. Carmona, Exponential localization in one dimensional disordered systems, Duke Math. J. 49 (1982), 191-213.

3. F. Delyon, H. Kunz and B. Souillard, One dimensional wave equations in disordered media, Ecole Polytechnique preprint.

4. I. Goldsheid, S. Molchanov And L. Pastur, A pure point spectrum of the stochastic one dimensional Schrödinger equation, Func. Anal. Appl. 11 (1977), 1-10.

5. M. HeRmann, A method for majorizing the Lyaponov exponent and several examples showing the local character of a theorem of Arnold and Moser on the torus of dimension 2, Ecole Polytechnique preprint.

6. R. JoHNSON, Lyaponov exponents for the almost periodic Schrödinger equation, U.S.C. preprint. 
7. R. Johnson AND J. Moser, The rotation number for almost periodic potentials, Comm. Math. Phys. 84 (1982), 403-438.

8. H. KUnZ AND B. SouIllaRd, On the spectrum of random finite difference operators, Comm, Math. Phys. 76 (1980), 201-246.

9. S. Molchanov, The structure of eigenfunctions of one dimensional unordered structures, Math. USSR Izv. 12 (1978), 69-101.

10. R. NeVAnlinna, Analytic Functions, Springer, 1970.

11. V. I. Osceledec, A multiplicative ergodic theorem. Lyaponov exponents for dynamical systems, Trudy Mosk. Mat. Obsc. 19 (1968), 679.

12. B. Simon, Almost periodic Schrödinger operators: A review, Adv. Appl. Math., to appear.

13. D. Thouless, $A$ relation between the density of states and range of localization for one dimensional random systems, J. Phys. C5 (1972), 77-81.

14. B. Aupetit, Proprietes spectrales des algebres de Banach, Springer Lecture Notes in Mathematics, $735,1979$.

15. J. D. Newburgh, The variation of spectra, Duke Math. J. 18 (1951), 165-176.

16. E. VESENTINI, On the subharmonicity of the spectral radium, Boll. Un. Mat. Ital. 4 (1968), 427-429.

Department of Mathematics, California institute of Technology, Pasadena, California 91125.

Simon also at Department of Physics. 